\title{
International Session 2
}

\section{Re-operation cases of the Allergic fungal sinusitis}

\author{
Kiyoshi Yanagi
}

ENT department

The allergic fungal sinusitis (AFS) is different from the eosinophilic sinusitis in its clinical conditions. Especially the prognosis is most characteristic of this. That is, the AFS recovers perfectly, but the eosinophilic sinusitis does not recover perfectly.

Also, the eosinophilic sinusitis recurs repeatedly. But AFS recovers almost perfectly after 2 years of the operation. This time, we considered 3 cases of AFS which were needed to perform re-operation for the purpose of elucidating the reason. I thought an operation different from a usual paranasal sinusitis should be chosen when the result AFS was doubted.

First, AFS develops in unilateral side, but Eosinophilic sinusitis develops in bilateral side.

And an affected side is the side where ventilation failures have occurred. Or the narrow side by in convex side of the septal deviation or hypertrophic concha bullosa.

Still now, the operations were performed reconstructions of the nasal septum and opening of the affected sinus cavity in the AFS. In our experiences, 8 cases in 12 cases were healed within 2 years after these operations. But even if more than 2 years pass after an operation, the remaining 4 cases are a repeating recurrence. These cases recurred in a different paranasal sinus from the first time. That suggests either different paranasal sinus in the same side or the opposite side's paranasal sinuses. This means that AFS shifts to another paranasal sinus. Also, the opening of the first time operation was insufficient for the paranasal sinus which recurred. And I thought the opposite paranasal sinuses which have no pathological changes in the first operation should also consider opening. 